\title{
Laboratório Virtual de Aprendizagem: Uma Proposta Taxonômica
}

\author{
Érico M. H. Amaral ${ }^{1,2}$, Bárbara Ávila ${ }^{2}$, Herik Zednik², Liane Tarouco ${ }^{2}$ \\ ${ }^{1}$ Instituto Federal Sul-rio-grandense - Campus Santana do Livramento \\ ${ }^{2}$ Programa de Pós-graduação em Informática na Educação - Universidade Federal do \\ Rio Grande do Sul (UFRGS) \\ ericohoffamaral@gmail.com, barbaragorzizaavila@gmail.com, \\ hzednik@hotmail.com, liane@penta.ufrgs.br
}

\begin{abstract}
Resumo. Este artigo apresenta uma proposta de taxonomia para Laboratórios Virtuais de Aprendizagem. A classificação é composta por duas dimensões nas quais busca-se analisar o nivel de interatividade de um Laboratório Virtual, bem como o nivel de suporte oferecido pela arquitetura aplicada. $O$ embasamento teórico se alicerçou em Schwier e Misanchuk para definir os niveis de interatividade e em Santos para distinguir os tipos de arquitetura de software. Inicialmente é abordado o conceito de laboratórios de aprendizagem e seus modelos, com foco nos Laboratórios Virtuais de Aprendizagem (LVA). Após, descreve-se a taxonomia proposta e aplica-se a mesma na avaliação de dois LVA com a finalidade de validá-la. O trabalho se justifica pela necessidade de orientação aos elaboradores de LVA e compreensão dos usuários acerca do assunto.
\end{abstract}

PaLVAras-chaves: Laboratório Virtual. Taxonomia. Interatividade.

Abstract. This article proposes a taxonomy for Virtual Learning Labs. Trough the two-dimensional classification we seek to analyze the Virtual Labs levels of interactivity, as well as the levels of support offered by the architectures implemented. The theoretical foundations are built upon Misanchuk and Schwier as to define the levels of interactivity and upon Santos as to distinguish the types of software architectures. Initially it is discussed the concept of learning labs and their models, focusing on Virtual Learning Labs (LVA). Then, the proposed taxonomy is described and the same applies to the evaluation of two LVAs in order to validate it. This research is justified by the need for guidance from developers of LVA and understanding on the subject from users.

Keywords: Virtual Lab. Taxonomy. Interactivity.

\section{Introdução}

Os estudos e avanços das Tecnologias de Informação e Comunicação (TIC) denotam atualmente uma nova concepção e foco na construção de recursos disponibilizados por tais tecnologias no âmbito educacional. Assim as TIC têm contribuído de maneira significativa para o redimensionamento das estratégias de ensinar e aprender, proporcionando diferentes ferramentas e artefatos para o apoio ao processo de aprendizagem. 
O uso de laboratórios de aprendizagem tem se tornado mais frequente e essencial em atividades educacionais. Essa estratégia se consolida entre os educadores por proporcionar um espaço onde os estudantes podem experimentar diversas situações úteis ao seu desenvolvimento. Assim, aprender fazendo é uma expressão que se revela em atividades laboratoriais e se fortalece através da Teoria Construtivista por considerar que o conhecimento deve ser construído pelo aprendiz, através da interação com o objeto e não transmitido pelo professor.

Este estudo pretende apresentar uma taxonomia para Laboratórios Virtuais de Aprendizagem (LVA), fazendo uso das variáveis de interação e arquitetura para representar a classificação proposta. Desta forma, as estratégias e recursos utilizados poderão ser analisados conforme estas duas variáveis, de modo que interajam, mas, por sua vez sejam relativamente independentes.

Os pontos principais que se estabelecem neste trabalho são: introdução sobre o conceito de Laboratórios de Aprendizagem, com seus respectivos modelos, dando ênfase aos Laboratórios Virtuais; apresentação da taxonomia para laboratório de aprendizagem virtual e aplicação da mesma em laboratórios distintos para fins de validação; finalmente, na seção 4, apresentamos as considerações finais enfatizando a importância desse trabalho para desenvolvedores de laboratórios virtuais, designers instrucionais, professores e alunos.

\section{Laboratórios de Aprendizagem}

Um Laboratório de Aprendizagem tem como objetivo principal complementar a construção do conhecimento do estudante, por meio do reconhecimento explícito dos processos envolvidos na atividade de ensino (Corrêa et al. 2001). Tanto que, não apenas na área de ciências se verifica o uso de laboratório, ele vem alcançando expressivo destaque em áreas como psicologia, educação, artes, linguística, publicidade, entre outras.

Diversos estudos defendem a eficácia dos laboratórios nas atividades educacionais. Kerr (1964) relacionou os objetivos que podem ser compreendidos através do uso desse recurso. São eles: encorajar a observação e descrição acurada; promover métodos científicos de pensamento; desenvolver habilidades de manipulação; treinar na solução de problemas; preparar os estudantes para exames práticos; elucidar o aprendizado da teoria; verificar fatos e princípios; desenvolver métodos de investigação; despertar o interesse; e tornar os fatos mais reais. Nas últimas décadas outros trabalhos salientaram a importância dessa ferramenta. Johnstone e Al-Shuaili (2001) apontam que as tarefas de laboratório podem possibilitar o desenvolvimento de habilidades manipulativas, observacionais, interpretação de dados experimentais e de planejamento de experimentos.

\subsection{Tipos de Laboratórios de Aprendizagem}

Os laboratórios apresentam-se em diferentes modalidades, promovendo situações de ensino e aprendizagem dentro de contextos educacionais específicos. Schimidt e Tarouco (2008) apontam três modelos de laboratórios, sendo eles: presenciais, remotos e virtuais.

Os laboratórios presenciais consistem em espaços localizados na própria instituição de ensino, nos quais o aluno dispõe de artefatos para a realização de seus experimentos. Salienta-se que nos laboratórios presenciais é comum o aluno estar acompanhado do docente e dos colegas, havendo revezamento no uso do material, visto 
que a falta de recursos financeiros muitas vezes inviabiliza que cada aluno disponha de todo o conjunto de instrumentos e materiais necessários às suas experiências.

Já nos laboratórios remotos, não é necessária a presença do aluno na instituição para que este possa realizar seus experimentos. Este tipo de ferramenta oferece ao aluno uma interface que the permite à manipulação a distância de instrumentos. Conforme discutem Callaghan et al. (2008) e Ramos-Paja, Scarpetta e Martínez-Salamero (2010), é importante salientar que os estudantes nem sempre dispõem de um acesso direto a laboratórios para realizar seus experimentos, embora seja muito importante que os mesmos desenvolvam atividades práticas para que se apropriem do conhecimento necessário a sua futura prática profissional. Desse modo, dispor de um acesso remoto ao laboratório da instituição de ensino pode ser uma ótima opção para aqueles alunos que não têm condições de se deslocarem até o laboratório presencial.

A entrada nos laboratórios remotos exige, de um modo geral, uma autenticação do aluno e requerimento por parte do mesmo para o acesso aos instrumentos e experimento desejados. Após a autenticação, os indivíduos estão aptos a completar experimentos nas estações remotas de trabalho. Como exemplo para este tipo de laboratório pode-se destacar o projeto Diesel, o qual evoluiu de um laboratório onde os alunos tinham acesso isoladamente, a um espaço colaborativo no qual os alunos interagiam em uma mesma experiência, utilizando-se de recursos de áudio e vídeo, o que veio a promover uma maior sensação de proximidade do grupo durante a condução dos experimentos (Callaghan et al., 2007).

Nos LVA os alunos interagem com representações virtuais que reproduzem o ambiente de um laboratório real. Este tipo de aplicação é totalmente baseado em simulações, dispondo somente de representações computacionais da realidade. Por não haver uma limitação em termos de número de instrumentos disponíveis nesta modalidade, não há necessariamente uma obrigatoriedade de autenticação por parte do aluno, nem tampouco é necessária a reserva de horário para uso do laboratório virtual, sendo este acessado a qualquer momento, sem maiores restrições.

Um exemplo de LVA é o Laboratório Virtual de Matemática pertencente à Universidade Regional Unijuí. Este laboratório dispõe de um vasto conjunto de atividades voltadas para a educação básica, ensino fundamental e médio. Nas experiências disponibilizadas pela aplicação os alunos trabalham com a alteração de parâmetros e também com a manipulação de objetos em diferentes situações envolvendo geometria, álgebra, trigonometria e outras áreas da matemática. Os estudantes iniciam o processo de aprendizagem a partir do seu contato com os conceitos de matemática, abordados em tarefas que propõem e guiam o aluno em uma série de experiências.

Ainda pode-se citar o Laboratório Virtual de Química, desenvolvido por Dalgarno et al. (2009) com a finalidade de preparar os discentes para atuação em laboratórios presenciais, estimulando seu conhecimento sobre os artefatos a serem utilizados nas atividades laboratoriais.

\subsection{Laboratórios Virtuais de Aprendizagem}

Os LVA consistem em plataformas digitais oferecidas com o intuito de dar suporte à realização de experiências sem a necessidade da presença do usuário em um determinado local, tal como ocorre no contexto dos laboratórios reais. Iowa (1999, p. 10) define os LVA como "[...] espaços eletrônicos de trabalho destinados à colaboração a distância e experimentação em pesquisa ou outra atividade criativa para gerar e distribuir resultados utilizando a informação distribuída e as tecnologias de comunicação". 
Um laboratório digital promove o acesso a experimentos a partir de um espaço virtual, compensando a falta de interação e a indisponibilidade de horários ou de recursos necessários às experiências práticas.

Iowa (1999) argumenta que o LVA não deve ser visto como um substituto do laboratório real, mas sim como uma ferramenta complementar capaz de gerar novas oportunidades em situações comumente dificultadas por questões financeiras, quando no contexto de uso dos laboratórios reais.

Um fator relevante que dá ênfase à necessidade de uso dos laboratórios digitais como forma de complementar as atividades práticas é justamente a questão da dificuldade de acesso, que muitos estudantes enfrentam, para estarem presencialmente em sua instituição de ensino, como ocorre no caso dos alunos da Educação a Distância (EAD). Pelo fato da grande maioria do alunos da EAD estarem alocados em pólos distantes do seu município de origem, o deslocamento à instituição se dá em momentos específicos, pré-determinados e que buscam minimizar o desgaste do aluno com viagens que possam tornar inviável a sua permanência no curso. Neste contexto, as atividades de laboratório ocorreriam numa frequência muito baixa, se a instituição não disponibilizasse um Laboratório Virtual onde todos os alunos, independentemente de sua localização geográfica tivessem a oportunidade de acesso.

Em áreas do conhecimento que demandam muitas atividades práticas, como a engenharia, a química ou a física o uso do laboratório é um componente essencial do currículo. Além disso, Callaghan et al (2008) salientam que a combinação da teoria com as atividades práticas é um elemento de suma importância para que ocorra a aprendizagem significativa. Os mesmos autores defendem o uso de laboratórios digitais com estudantes a distância do curso de engenharia sob a argumentação de que a web somada a estratégias de aprendizagem possibilitam métodos realísticos nas experiências práticas " [...] permitindo que estudantes alocados remotamente desenvolvam suas atividades de laboratório, transpondo limitações geográficas e temporais, ao mesmo tempo em que são desenvolvidas habilidades no uso de instrumentos e sistemas reais (Callaghan et al., 2008)".

A possibilidade de experimentação prática nos laboratórios virtuais é um elemento essencial à eficácia destas plataformas, no que tange à sua função educacional. Dalgarno et al. (2009) confirmam esta constatação em sua pesquisa realizada com o uso de um laboratório virtual como meio de preparação dos estudantes para as sessões em laboratório real. O LVA proposto pelos autores consistia somente numa réplica do espaço real, porém sem a possibilidade de realização de quaisquer simulações. Questionários e entrevistas posteriores realizados com os estudantes que tiveram ou não contato com o Laboratório Virtual apontaram que a maior fonte de ansiedade dos alunos, com relação às atividades no laboratório presencial, ocorria em torno do domínio conceitual necessário à realização dos experimentos. O Laboratório Virtual, neste caso, sem a realização de experiências, não veio a contribuir para a redução da ansiedade dos cursistas. A maioria dos alunos manifestou que o LVA seria mais eficiente se tivesse proporcionado meios de realização de experiências prévias às sessões de laboratório. Concorda-se plenamente com a posição dos alunos em relação à finalidade do LVA, pois se percebe a possibilidade de experimentação como a função fundamental de qualquer tipo de laboratório de aprendizagem.

\section{Taxonomia para Laboratórios Virtuais de Aprendi zagem}

Uma vez introduzido o LVA como recurso pedagógico no contexto educacional, esta ferramenta poderá contribuir para auxiliar as intervenções do professor e favorecer a autonomia dos alunos, estimulando-os na construção de conhecimentos significativos. 
Assim, o LVA necessita ser interativo, ergonômico, sólido quanto ao conteúdo abordado e eficiente quanto à organização lógica do funcionamento do sistema computacional.

Os LVA vêm sendo amplamente utilizados nos mais diversos níveis e modalidades de ensino e apresentam características bastante variadas, surgindo assim a necessidade de classificação para orientação dos usuários e elaboradores.

Pensando nesses fatores, esse estudo desenvolveu uma taxonomia voltada para os LVA a partir de duas variáveis, o nível de interatividade do sistema e seus critérios arquitetônicos. Como mostra a figura 01 .

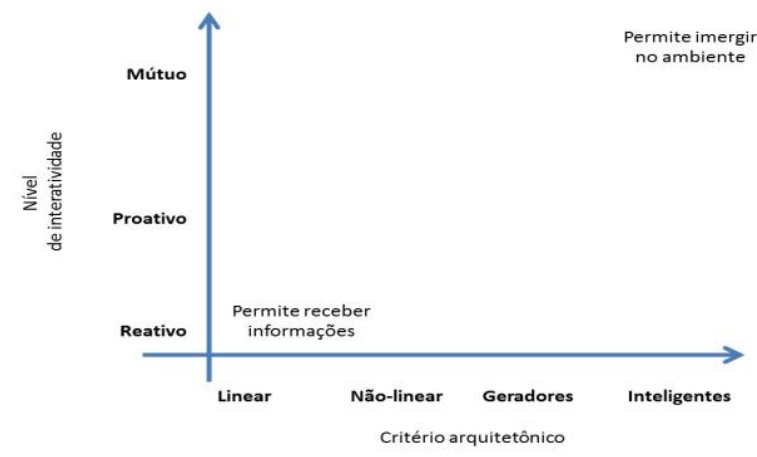

Figura 01 - Representação gráfica da taxonomia Fonte: Próprio autor

O eixo horizontal, conforme a Figura 01, apresenta a organização arquitetônica em torno da estrutura do LVA. Essa dimensão refere-se à maneira como o LVA é programado para oferecer determinados recursos: quanto mais se aproxima do pólo de Inteligentes, mais dispõe de recursos que facilitarão a interação do usuário; inversamente, quanto mais se aproxima do pólo Linear, menos suporte é oferecido e mais limitado é o ambiente.

O eixo vertical remete ao tipo de interação proporcionada pelo LVA. Neste caso, quanto mais próximo estiver do polo Mútuo, mais interatividade é disponibilizada ao usuário. Ambientes com menor nível de interação aproximam o usuário do pólo Reativo.

\subsection{Critério Arquitetônico}

O Critério Arquitetônico no contexto desta pesquisa descreve a quantidade de recursos disponibilizados pelo software do LVA. A variável relacionada à arquitetura foi adaptada para o objeto de estudo a partir dos trabalhos de Gilberto Santos (2001), que desenvolveu uma classificação voltada para softwares, o qual dividiu em quatro tipos distintos: linear, ramificado, gerador e inteligente.

A arquitetura linear, embora surgida na década de 50, ainda é bastante presente na concepção de muitos softwares. Influenciados pela teoria behaviorista de Skinner, o estímulo-resposta é a base do seu funcionamento. "O educando deve partir de um determinado ponto e chegar a um objetivo final preestabelecido. A avaliação é indicada através do número de acertos/erros e o reforço significa a repetição, porém respeita-se o ritmo de aprendizagem do educando (Santos, 2001)". O conhecimento é apresentado ao estudante em uma determinada ordem, seguindo uma sequência estabelecida pelo programador. Nesse tipo de arquitetura, o maior problema é a falta de estímulo ao raciocínio do estudante quando exposto a diferentes situações.

Os softwares não-lineares (ou Ramificados) apresentam '[...] uma configuração mais complexa e avalia o aluno no ponto de entrada para a partir dessa avaliação conduzi-lo por caminhos de aprendizagem em função dos conhecimentos detectados" 
(Santos, 2001). Este tipo de software apresenta programação em forma de árvore, o que possibilita feedback ao usuário, e são baseados na concepção de que os estudantes apresentam perfis intelectuais singulares que, por este motivo, deve ser adaptado para situações de aprendizagem diferenciadas. Uma característica típica dos softwares ramificados é o fato de estarem centrados no professor, ou seja, o papel do aluno ainda é passivo e se resume a responder alguma questão, após compreensão do conteúdo.

Os sistemas gerativos, também chamados de sistemas adaptativos, surgiram no final da década de 60. Foram "elaborados tendo como base estratégias tutoriais geradoras, o software é configurado em forma de árvore, dando múltiplas possibilidades de interação educando-máquina" (Santos, 2001). Este tipo de aplicação permite a geração de problemas, fazendo relação com o nível de conhecimento do aluno, construindo a solução e diagnosticando a resposta do estudante. O problema dos softwares geradores encontra-se no fato de não serem adequados para todo tipo de domínio de ensino.

Os sistemas inteligentes representam um aperfeiçoamento sobre o modelo geradores, e está fundamentado nos princípios da inteligência artificial. "Nesse tipo de sistema, o computador é capaz de identificar graus de complexidade, diagnosticar, controlar, prever, conceber, planificar, monitorar e instruir" (Santos, 2001). Para um sistema ser considerado inteligente, Jonassen (1993) apud Gavidia e Andrade (2003) destacou três pontos:

Conteúdo do tema ou especialidade deve ser codificado de modo que o sistema possa acessar as informações, fazer inferências ou resolver problemas; sistema deve ser capaz de avaliar a aquisição deste conhecimento pelo aluno; as estratégias tutoriais devem ser projetadas para reduzir a discrepância entre o conhecimento do especialista e o conhecimento do aluno.

Os sistemas inteligentes se destacam por possibilitar uma sequência de ensino que não é determinada pelo desenvolvedor ou pelo designer instrucional, além de favorecer a comunicação entre tutor-aluno, proporcionar a aprendizagem colaborativa, permitir diagnósticos mais particularizados e detalhados aos alunos, possibilitam simuladores cada vez mais próximos da realidade (numa experiência, por exemplo, o aluno pode operar com múltiplas variáveis e manipular os mesmos elementos de uma experiência real a fim de obter os mesmos resultados) e há a possibilidade de agregar a realidade virtual. Outro aspecto importante é que a interação pode ser bastante diversificada, transitando desde o nível mais reativo ao mútuo, ou seja, por meio de padrões que esperam o aluno realizar uma ação; aos que observam o aluno ao executar uma tarefa, faz intervenções, explicam um conceito quando percebem a necessidade do estudante ou quando solicitado. Ressaltamos ainda, a possibilidade de avaliação ao longo de todo o processo de ensino/aprendizado, acompanhando a evolução do discente e suas dificuldades.

\subsection{Nível de Interatividade}

O conceito interatividade remete a qualquer atividade, objeto ou sistema caracterizado pela ativa participação de um usuário, durante a sua realização ou funcionamento. Várias formas de interatividade podem ser encontradas em um ambiente de ensinoaprendizagem: desde o contato do aluno com o professor até as relações entre os próprios alunos ou entre alunos e o sistema, pois segundo Moura Filho e Oliveira (1999) a qualidade de um processo educacional está calcada sobre um padrão de comunicação contínua e bidirecional entre as partes envolvidas. 
Segundo Schwier e Misanchuk (1994), a interatividade de um determinado sistema pode ser classificada de acordo com 4 diferentes níveis: nível 1, caracterizado por uma baixa interatividade; o nível 2 é identificado por um grau intermediário de interação; um alto grau de interatividade é identificado no nível 3 por meio da união de diferentes mídias; e o nível 4 é definido pela união de todas mídias disponíveis em um único ambiente, com o domínio de inovações futuras.

O eixo "Nivel de Interatividade" da Figura 01, proposta neste estudo, está baseado na taxonomia descritiva de interação indicada por Schwier e Misanchuk (1994). Neste contexto são enumerados os tipos de interações constantes em cada nível, a fim de relacionar os laboratórios virtuais com alguns índices de interatividade e classificá-los de tal forma. O quadro 01, mostra a taxonomia de interação revisada, baseada em 3 níveis ordenados, de acordo com a qualidade institucional da interação.

\section{Quadro 01 - Taxonomia de interação revisada (SCHWIER e MISANCHUK, 1994)}

\begin{tabular}{|c|c|c|}
\hline Niveis & Funções & Transações \\
\hline Reativo & Confirmação & Barra de espaço/tecla entrada \\
\hline Proativo & Ritmo & Meta tela tátil \\
\hline Mútuo & Navegação & Tela tátil rastreador de raio \\
\hline \multirow{4}{*}{} & Requisição & Mouse com clique \\
\cline { 2 - 3 } & Elaboração & Mouse para arrastar \\
\cline { 2 - 3 } & & Código de barras \\
\cline { 3 - 3 } & & Teclado-resposta \\
\cline { 3 - 3 } & & Teclado-construção \\
\cline { 3 - 3 } & & Entrada de voz \\
\cline { 3 - 3 } & & Interface de realidade virtual \\
\cline { 3 - 3 } & & \\
& & \\
\cline { 3 - 3 } & & \\
& & \\
\end{tabular}

Para o entendimento do quadro 01, tem-se a interação reativa como uma resposta ao estímulo do sistema sobre o usuário, ou resposta para a questão colocada. Neste contexto o material educacional guia o aluno, através de um fluxo pré-determinado, durante toda a realização dos procedimentos de uma determinada tarefa. $\mathrm{Na}$ interação proativa o aluno vai além, selecionando ou respondendo as estruturas existentes, construindo assim situações únicas e elaborações que forçam os limites do sistema. Por fim, a interatividade mútua se caracteriza por dispor de recursos de inteligência artificial ou realidade virtual, onde o aluno imerge totalmente no ambiente disponibilizado pela aplicação, sendo estes sistemas mutuamente adaptativos.

\section{Validação da taxonomia proposta}

Com a proposta da taxonomia aprimorada, conforme a Figura 02, pretende-se alcançar um nível de entendimento lógico, baseado em uma matriz de pontos $5 \times 7$ que permite a classificação de diferentes LVAs de acordo com o seu nível de interatividade e sua composição estrutural. 


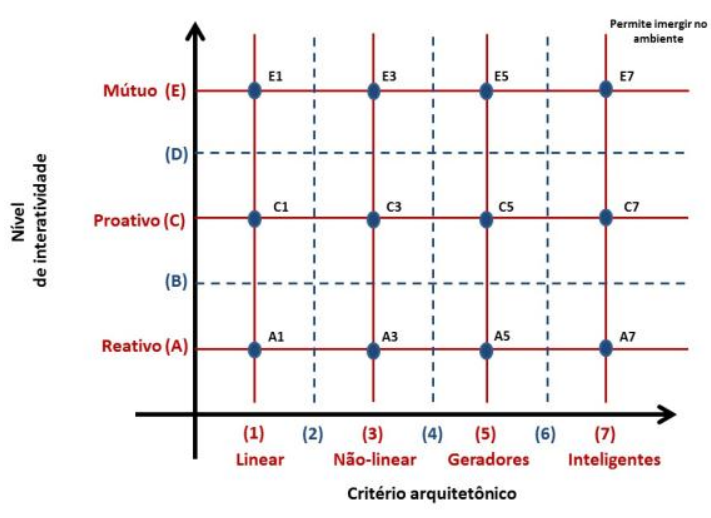

Figura 02 - Matriz de Classificação -Taxonomia proposta



Figura 03 - Fronteira de classificação Fonte: Próprio autor

Fonte: Próprio autor

Neste método de classificação, os LVA são avaliados a partir de duas dimensões as quais definem seu nível de interatividade e a complexidade de sua arquitetura. A avaliação é realizada através do cruzamento entre os eixos horizontal e vertical da Figura 01, compondo a matriz apresentada na Figura 02. Por meio da estrutura desta matriz é possível visualizar a taxonomia proposta e utilizar os seus pontos para a categorização dos laboratórios virtuais.

A Figura 03 apresenta os limites identificados para classificar o nível de interatividade relacionado à estrutura disponibilizada pelo LVA. A partir desta, é plausível afirmar que uma aplicação de estrutura linear permite apenas uma interatividade Reativa, devido à quantidade reduzida de recursos disponibilizados pela mesma. A linha vermelha nesta Figura demonstra a fronteira de interatividade imposta pelos critérios arquitetônicos.

Desta forma, um laboratório que apresente recursos como: mural, chat, fórum, wiki, blogs, envio de sugestões, workgroup virtual e ainda, ofereça feedback do sistema após a conclusão das experiências virtuais, poderíamos classificá-lo, de acordo com a Figura 03, como um LVA 3.3 (Gerador-Mútuo). A classificação na matriz proposta não é rígida, de modo que um LVA pode se situar entre dois níveis distintos em uma mesma dimensão, apresentando características de ambos.

Para validar a taxonomia proposta, foram avaliados dois laboratórios virtuais, apresentados previamente na seção 2 , um na área de matemática e outro de química.

\subsection{Laboratório Virtual de Matemática}

O Laboratório Virtual de Matemática (Figura 04), pertencente à Universidade Regional Unijuí, dispõe de um vasto conteúdo, distribuído entre as áreas da Educação Básica, Ensino Fundamental e Médio. 


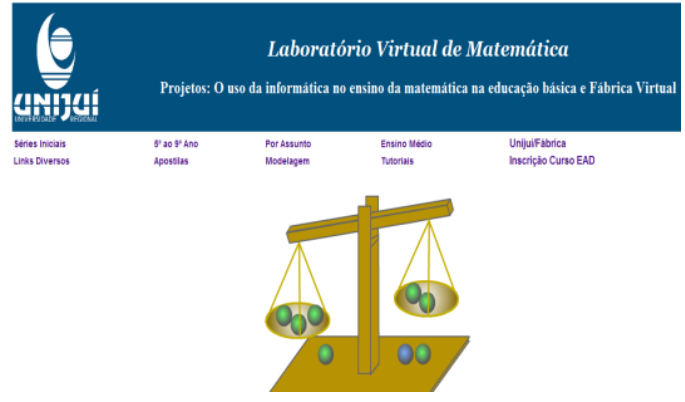

Figura 04 - Laboratório Virtual de Matemática

Fonte: http://www.projetos.unijui.edu.br/matema

O acesso ao laboratório é totalmente livre, e não há nenhum tipo de acompanhamento sobre o desempenho dos usuários, o que não oportuniza o nível de Interatividade Mútua. Devido à liberdade que o usuário tem em sua navegação, assim como no que diz respeito às possibilidades de alteração de valores e manipulação de objetos, pode-se classificar o nível de interatividade deste LVA como Proativo.

Em se tratando dos critérios arquitetônicos, o Laboratório Virtual de Matemática encontra-se no intervalo entre a Linearidade e a Ramificação. Pode-se considerar que o LVA apresenta características da Linearidade pelo fato de que o objetivo de cada atividade é predefinido e os experimentos são realizados de acordo com instruções preestabelecidas pelo sistema. Por outro lado, ocorre uma aproximação com a Ramificação devido às possibilidades de navegação de que o usuário dispõe. Ou seja, não há uma sequência imposta ao usuário, o qual pode optar por diferentes experimentos a realizar em seu processo de aprendizagem. Desse modo, de acordo com a taxonômica proposta, este laboratório classifica-se no tipo $\mathrm{C} 2$ da matriz, exposta na Figura 05.

\subsection{Laboratório Virtual de Química}

O laboratório de química desenvolvido na Universidade Charles Sturt (Figura 06) também apresentou-se como uma ferramenta de acesso livre aos estudantes que participaram da pesquisa. $\mathrm{O}$ acesso a este LVA ocorre por meio de um CD-Room não havendo formas de controle de acesso ou de desempenho dos cursistas ao longo das atividades.



Figura 06 - Laboratório Virtual de Química Fonte: Dalgarno et al. (2009)

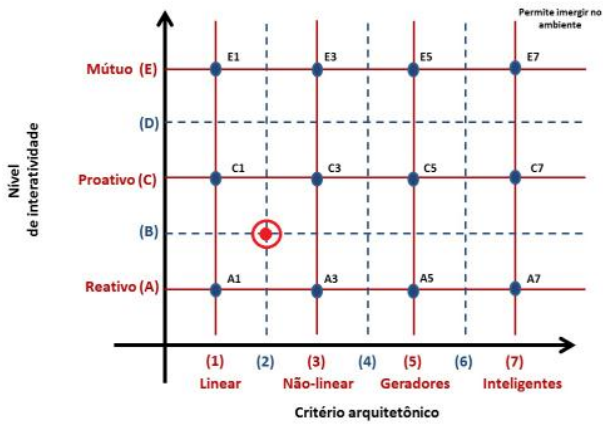

Figura 07 - Taxonomia do Laboratório Virtual de Química

No que se refere à interatividade deste laboratório avalia-se que o mesmo apresenta características que se estendem entre os níveis Reativo e Proativo. O sistema aproxima-se do conceito de Reativo pelo fato de que o mesmo foi idealizado somente 
para guiar $\mathrm{o}$ aluno nas atividades presenciais de laboratório, sem oferecer a possibilidade de ações criativas por parte dos cursistas. Por outro lado, o LVA apresenta características proativas, já que não impõe ao usuário uma navegação guiada. Ou seja, há liberdade de escolha nos espaços a serem explorados dentro do laboratório, embora o cursista não tenha a liberdade de realizar seus experimentos dentro do laboratório.

Em relação aos critérios arquitetônicos, o LVA, assim como o anterior apresentase no intervalo entre os níveis Linear e Ramificado. Tal como o nível Ramificado, o LVA apresenta diversos caminhos a serem seguidos pelo cursista. Por outro lado, o mesmo não atinge totalmente este nível, pois não ocorre uma pré-avaliação do aluno em sua entrada no ambiente para a posterior adequação de suas atividades. A partir desta avaliação, constata-se que na matriz da Figura 07, este laboratório classifica-se no tipo B2.

\section{Considerações finais}

A evolução das tecnologias de ensino mostra que a utilização de recursos computacionais como ferramentas efetivas para aprendizagem hoje é uma realidade. Esta pesquisa realizou um estudo sobre os Laboratórios de Aprendizagem Virtuais, analisando as estratégias didáticas adotadas nestes ambientes a fim de decompor seu funcionamento e avaliar suas características a partir de variáveis simples. No contexto da construção do conhecimento pelo aluno, identificou-se que alguns fatores para a utilização didática eficiente de um LVA estariam relacionados ao nível de interatividade proporcionado pela estrutura da aplicação. Deste modo, foi possível elaborar uma proposta de taxonomia baseada em dois eixos: nível de interatividade e critério arquitetônico, os quais variam de acordo com a possibilidade de recursos oferecidos ao usuário.

Para validação da proposta foram analisados dois laboratórios de aprendizagem, o que permitiu consolidar a taxonomia em distintas áreas de ensino. O resultado desta experiência demonstrou a eficiência da matriz de classificação implementada. A taxonomia resultante deste trabalho servirá de base para futuras avaliações de LVA para que estes possam se firmar como espaços mais interativos, pedagógicos e com arquiteturas eficientes e eficazes.

\section{Referências}

GAVIDIA, Jorge Juan Z.; ANDRADE, Leila Cristina V. Sistemas tutores inteligentes. Universidade Federal do Rio de Janeiro: Rio de janeiro, 1993

CALlAGHAN, Michael, J.; HARKIN, Jim; MCGINNITY, Thomas M.; MAGUIRE, Lion $P$. Client-se rver architecture for collaborative remote experimentation. Journal of Network and Computer Applications, Northland, v. 30, p. 1295-1308, set/2007.

CALLAGHAN, Michael, J.; HARKIN, Jim; MCGINNITY, Thomas M.; MAGUIRE, Lion P. Intelligent User Supportin Autonomous Remote Experimentation Environments. 2008. Trabalho apresentado em IEE Transation Industrial Eletronics, 2008.

CORRÊA, Geny D.; CORRÊA, Carlos J.; SANTOS, Víctor B. O Laboratório de aprendizagem e a reconstrução do conhecimento. Congresso Brasileiro de Engenharia - COBENGE, 2001.

DALGARNO, Barney; BISHOP, Andrea G.; ADLONG, Willian; BEDGOOD JR., Danny R. Effectiveness of a Virtual Laboratory as a preparatory resource for 
Distance Education chemistry students. Computers \& Education, v. 53, p. 853-865, Mai/2009.

IOWA, Ames. Report of the Expert Meeting on Virtual Laboratories . ParisÇ Unesco. 2000.

JOHNSTONE, A. H; AL-SHUAILI, A. Leaming in the laboratoty: some thoughts from the literature. University Chemistry Education, $\mathrm{n}^{0}$ 5, 2001.

KERR, John F. Pratical work in school science: an account of an inquiry into the nature and purpose of pratical work in school science in England an Wales. Leicester: Leicester University press, 1964.

MOURA FILHO, César OLVAo de; OLIVEIRA, Mauro. Videoconferência em educação a distância. Fortaleza, CE: CEFET, 1999.

NITZKE, J. A, FRANCO, S. R. Aprendizagem cooperativa: utopia ou possibilidade. Informática na Educação: teoria e prática, v. 5 n² 2, Porto Alegre, 2002.

RAMOS-PAJA, Andrés C.; SCARPETTA, José M. R.; MARTÍNEZ-SALAMERO, Luis. Integrated Learning Platformfor Intemet-Based ControlEngineeringEducation. 2010. Trabalho apresentado em IEE Transation Industrial Eletronics, 2010.

SANTOS, Gilberto Lacerda; VIDAL, Eloísa Maia; BESSA MAIA, Educação informática e professores. Fortaleza: Edições Demócrito Rocha, 2002.

SCHIMIDT, Marcelo A. R.; TAROUCO, Liane M. R. Metaversos e laboratórios virtuais - possibilidades e dificuldades. Revista de Novas Tecnologias na Educação, Porto Alegre, v. 6, n. 1, p 1-12, jul/2008.

SCHWIER, Richard A.; MISANCHUK, Earl R. Interactive Multimedia Instruction.

New Jersey: Englewood Cliffs, 1994. 